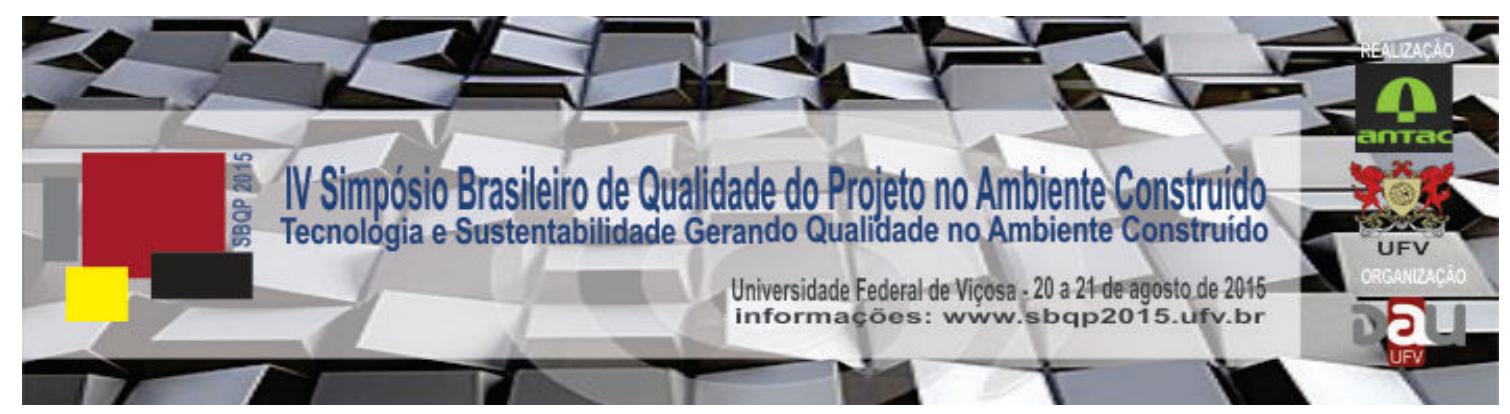

\title{
SUBSÍDIOS PARA A CRIAÇÃO DE UM SISTEMA DE INDICADORES DE DESEMPENHO EM EDIFICAÇÕES'
}

\author{
FRANÇA, Ana Judite Galbiatti Limongi \\ FAUUSP, alimongi@usp.br \\ ORNSTEIN, Sheila Walbe \\ FAUUSP, sheilawo@usp.br
}

\begin{abstract}
RESUMO
A gestão eficiente da informação da construção, ao longo das fases do ciclo de vida útil da edificação, pode possibilitar o aproveitamento dos resultados de diagnósticos realizados em edificações em uso ao processo de projeto. Para tal, é necessário que o desempenho aferido seja eficientemente associado a componentes, sistemas construtivos e ambientes de uma edificação, de forma a permitir uma avaliação sistemática. Os resultados devem ser consultados de modo assertivo, inclusive provendo subsídios para sua comparação entre edificações, necessária à elaboração de benchmarks. Para a avaliação de desempenho do ambiente construído, é oportuno estabelecer um sistema gestão da informação da construção que possibilite a análise e a comunicação dos resultados de forma estruturada. Esse artigo apresenta os resultados parciais de uma pesquisa de doutorado em andamento, que se propõe a identificar aspectos e métricas prioritários para a meta-avaliação do projeto em edificações de tipologia semelhante.
\end{abstract}

Palavras-chave: Desempenho no ambiente construído, Gestão da Informação da Construção, Avaliação Pós-Ocupação (APO).

\begin{abstract}
It is important to provide efficient management of building information, throughout all the stages of its life cycle. In this scenario, should be possible the use of the results of Post-Occupancy Evaluation (POE) diagnoses into the design process. For this, it is necessary that the assessment results are efficiently related to components, building systems and environments, in order to allow a systematic evaluation. For the evaluation of performance criteria compliance, it is appropriate to establish a construction information management system, in order to enable analysis and structured communication of building information. This article presents the partial results of a doctorate research, which aims to identify priority issues and metrics for the metaevaluation of buildings with the same typology, so as to enable the development of benchmarks.
\end{abstract}

Keywords: Building Performance, Construction Information Management, PostOccupancy Evaluation (POE).

\footnotetext{
${ }^{1}$ Trabalho apresentado no IV SBQP 2015. Universidade Federal de Viçosa. Disponível em: doi> http://dx.doi.org/10.18540/2176-4549.6028
} 


\section{INTRODUÇÃO}

O ambiente construído consiste no resultado de um conjunto de sistemas e processos. Para sua produção e operação, se pressupõe a integração de equipes com diversas expertises e objetivos, tais como: projetistas, construtores, gestores e usuários. Dada esta complexidade, para que se possa controlar a qualidade no ambiente construído, é necessária a formalização dos procedimentos envolvidos e de suas interrelações (MELHADO, 1999).

Em se tratando de edificações com tipologia construtiva e programa de necessidades semelhantes, destaca-se a importância do aprendizado com a experiência pregressa, por meio da incorporação dos resultados obtidos referentes ao desempenho no ambiente construído em uso. Para sua avaliação, podem ser incorporados os métodos e técnicas de Avaliação PósOcupação (APO). Entretanto, para que se proporcione a efetiva realimentação de projeto, é necessário estruturar a informação de modo eficiente e sistemático, de modo a permitir o mapeamento claro entre os elementos, sistemas construtivos e ambientes e os resultados obtidos (FRANÇA, ORNSTEIN, 2014).

Além disso, diferentes responsáveis pelas decisões durante o processo de projeto e de construção de um empreendimento (stakeholders) podem ter entendimentos distintos quanto ao nível de prioridade de aspectos críticos (FEIFER, 2011). Sendo assim, é preciso tornar o processo decisório objetivo, por meio da adoção de instrumentos de gestão da qualidade do ambiente construído, como, por exemplo, sistemas de indicadores. Dentre as vantagens de sua adoção estão: o monitoramento do atendimento de metas e a identificação de aspectos relevantes para a elaboração de benchmarks, para a comparação entre edificações com características em comum.

\section{CONCEITOS E EXPERIÊNCIAS QUANTO À ADOÇÃO DE INDICADORES PARA A GESTÃO DA QUALIDADE}

Considerando que um indicador corresponde a determinada propriedade a ser mensurada e avaliada de modo a fornecer informações relevantes, relacionadas a determinado ambiente, a determinação de um sistema de indicadores deve possibilitar a avaliação mensurável, objetiva, rastreável e auditável das condições em determinado ambiente, inclusive permitindo uma análise do custo-benefício referente às ações a eles relacionadas (OECD, 1993).

Sistemas de indicadores vêm sendo adotados, nacional e internacionalmente, com o objetivo de avaliar tanto a qualidade do ambiente construído quanto das atividades da construção civil. Entende-se, nesse contexto, que qualidade corresponde ao nível de atendimento de um determinado produto a requisitos determinados (VAN DER VOORDT; VAN WEGEN, 2005).

Dentre os referenciais para a avaliação do desempenho de edificações, estão: Leadership in Energy and Environmental Design - LEED (UNITED STATES GREEN BUILDING COUNCIL, 2009) e Haute Qualité Environnementale - HQE (CERTIVÉA, 2012) e Building Research Establishment Environmental Assessment Methodology - BREAM (BRE, 2011), entidades estas que propõem práticas de gestão e determinam a adoção de indicadores, objetivando uma melhor 
qualidade ambiental da edificação. Embora os três exemplos mencionados se proponham a aferir a qualidade ambiental do ambiente construído, estes diferem na abordagem e na importância dada aos diferentes critérios de desempenho (SILVA, 2007; AULICINO, 2008; DEGANI, 2010, GALVÃO, 2012). No Brasil, O referencial AQUA (CARDOSO, 2007) apresenta critérios para a avaliação do desempenho ambiental, baseados nas diretrizes determinadas pelo referencial francês HQE (CERTIVÉA, 2012) e adaptados às necessidades e à realidade regional.

Os referenciais de certificação ambientais citados determinam, por meio da concessão de um certificado, uma maneira de comunicar aos envolvidos e à comunidade o nível de atendimento de um determinado empreendimento aos indicadores e processos por eles preconizados.

Também merecem análise as abordagens que se dedicam a identificar os indicadores-chave de desempenho (key performance indicators- KPIs), ou seja, métricas referentes ao desempenho de características construtivas e de operação - como, por exemplo, o consumo de energia (NEW BUILDINGS INSTITUTE, 2014). Tais indicadores podem ser utilizados para, entre outros objetivos, monitorar custos, avaliar o desempenho das ações de operação e de manutenção, avaliar a satisfação dos clientes e comparar desempenho entre diferentes projetos. Entretanto, o processo de avaliação deve ser consistente, mensurável e auditável (GALANEZ-ÁLVARES, 2013).

Sistemas de indicadores podem ser ainda consolidados com o objetivo de avaliar a qualidade e a produtividade das atividades de construção (COSTA et al., 2005). Também podem ser destinados à análise de um determinado aspecto de interesse. Labaki et al. (2007), por exemplo, propõem indicadores de eficiência energética para escolas estaduais em Campinas, por meio da análise, na fase de projeto, do desempenho de elementos construtivos no contexto do projeto, por meio de simulação computacional.

Por tanto, deve ser investigado o nível de atendimento de um projeto, produto ou ambiente construído aos objetivos pré-determinados. Sendo assim, é necessário definir instrumentos para a identificação de aspectos não previstos ou a serem melhorados, os quais devem fornecer dados que permitam a consolidação de um banco de dados para referência (VAN DER VOORDT; VAN WEGEN, 2005). Entretanto, os aspectos pertinentes à investigação irão variar em função dos objetivos específicos de um projeto ou um sistema de gestão. Considerando o contexto apresentado, a presente pesquisa propõe um sistema de indicadores que possam ser adotados para a avaliação do desempenho de edificações de tipologia padronizada em uso, bem como para a revisão das diretrizes de projeto adotadas para futuras construções e para a requalificação das existentes.

\section{MÉTODOS E TÉCNICAS}

A fase inicial da pesquisa foi dedicada à avaliação dos estudos de caso. $O$ universo determinado foi o de escolas estaduais paulistas, localizadas na Região Metropolitana de São Paulo, projetadas segundo tipologia padronizada e construídas a partir de 2003. Os resultados foram organizados em matrizes de diagnóstico (FRANÇA, ORNSTEIN, ONO, 2010) e sua tabulação permitiu a identificação de aspectos recorrentes àquele grupo de edificações. 
Também os resultados foram comunicados aos responsáveis pelo processo decisório na concepção de novas edificações de mesma tipologia, que, no caso em questão, corresponde ao corpo técnico e administrativo da Fundação para o Desenvolvimento da Educação (FDE).

O procedimento metodológico da pesquisa contemplou as seguintes etapas: avaliação de estudos de caso, de modo a prover resultados de levantamentos de campo, que demonstrem o comportamento de edificações em uso. Para esta fase, foram considerados os resultados obtidos por meio da aplicação de instrumentos de avaliação, roteiros de observações estruturadas (checklists) e medições dimensionais e de conforto ambiental, realizadas nas edificações.

Após o processamento das informações tabuladas, se deu uma fase de benchmarking, para a identificação de modelos de gestão da informação aplicáveis à prática da avaliação das edificações em uso. Este arcabouço teórico foi considerado como subsídio para a consolidação de um Sistema de Indicadores para a avaliação do desempenho do edifício quando em uso, de modo a permitir a incorporação, de modo sistemático, a realimentação de diretrizes.

\subsection{Conceitos e requisitos relacionados à AVALIAÇÃO de desempenho do ambiente construído}

É esperado que um edifício apresente características adequadas para atender às necessidades conscientes e inconscientes de seus usuários. Sendo assim, o desempenho do ambiente construído é definido como o atendimento a um conjunto de requisitos, pela edificação pronta e em uso. Entretanto, no Brasil, este conceito é, muitas vezes, erroneamente limitado à avaliação da qualidade dos sistemas e dos processos construtivos, em detrimento do resultado final (BORGES; SABATTINI, 2008).

Os critérios de desempenho e as definições de vida útil do edifício apresentados na norma brasileira NBR 15575:2013 (ABNT, 2013) são organizados conforme os seguintes grupos: habitabilidade (conforto higrotérmico, visual, tátil, acústico, antropométrico, acessibilidade, estanqueidade, condições de higiene), sustentabilidade (durabilidade, manutenibilidade e desempenho ambiental), segurança (estrutural, ao fogo e no uso). Estes critérios constituíram em balizadores para a elaboração das avaliações e diagnósticos da presente pesquisa. Os requisitos de desempenho de um conjunto arquitetônico, não se restringem ao comportamento de um determinado componente ou sistema construtivo. O desempenho efetivo de um ambiente é aferido considerando os desempenhos individuais dos componentes e serviços que o compõem e suas interações.

\subsection{Consolidação do sistema de indicadores}

Foram identificados três grupos de parâmetros, aplicáveis com objetivos distintos, a saber: avaliar o desempenho de componentes e sistemas construtivos; avaliar o nível de adequação dos ambientes de um empreendimento, considerando o uso previsto; e avaliar o desempenho global do empreendimento, frente a outras edificações com características semelhantes. Sendo assim, os indicadores são apresentados atribuídos aos componentes e sistemas construtivos ou globais (atribuídos ao ambiente ou ao 
empreendimento como um todo- de modo que possam ser identificadas as edificações de um conjunto, que apresentem melhor ou pior desempenho, sob determinado aspecto de interesse para, mediante a um aprofundamento da análise, isolar e identificar as variáveis responsáveis por tal condição).

Por meio da análise das matrizes de diagnóstico, constituídas a partir dos resultados da aplicação de APO aos estudos de caso e à luz das boas práticas determinadas pelos sistemas de gestão da qualidade ambiental (CARDOSO, 2007; DEGANI, 2010; GALVÃO, 2012), foram determinados os seguintes indicadores específicos, aplicáveis a componentes e sistemas construtivos: frequência de quebra ou dano (número de ocorrências/ total de unidades); número de não conformidades, quanto ao desempenho - a ser considerado por critério de desempenho pertinente (Figura 1) (ABNT, 2013).; frequência de medidas de manutenção preventiva (ocorrências/ total de unidades instaladas); frequência de medidas de manutenção corretiva (ocorrências/ por total de unidades instaladas); vida útil (vida útil efetiva/ vida útil prevista, em anos); custo (valor unitário/ unidade instalada); produção/instalação (total de unidades/ período de tempo); volume de resíduos gerado $\left(\mathrm{m}^{3} / \mathrm{ano}\right)$; disponibilidade de fornecedores homologados (total).

\section{Figura 1 - Número de não conformidades quanto ao desempenho (ABNT, 2013) identificadas nos estudos de caso objeto de aplicação de APO (FRANÇA, 2010)}

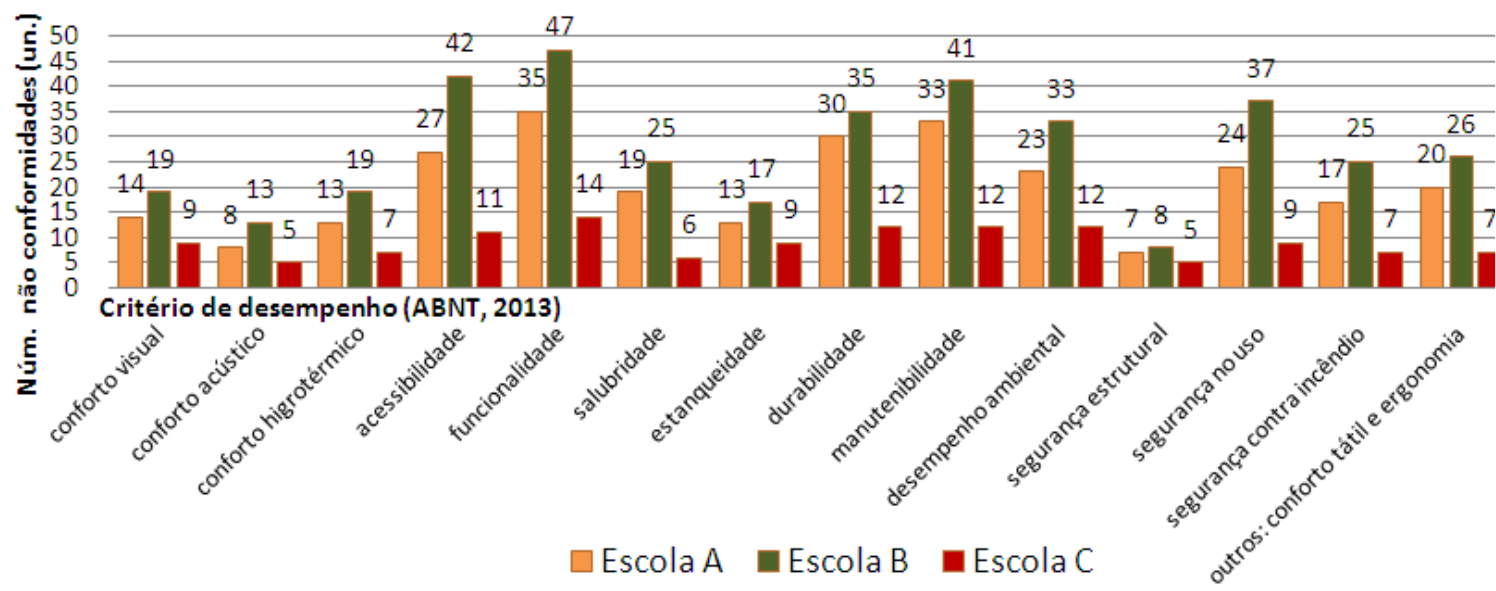

Fonte: acervo das pesquisadoras.

Considerando o desempenho esperado para o ambiente conforme o uso previsto, são elencados os seguintes parâmetros: nível de iluminância no plano de trabalho (LuX) (ABNT, 1992); nível de conforto acústico [dB(A)] (ABNT, 1987); nível de conforto térmico (total de horas/ano na zona de temperatura operativa aceitável) (ABNT, 2013); nível de adequação ao uso previsto (total de não conformidades/ total de ambientes com mesmo uso) (ABNT, 2013); taxa de renovação de ar (ANSI/ASHRAE, 2013a); Densidade de Potência Luminosa (W/m2) (ANSI/ASHRAE, 2013b).

Finalmente, considerando a edificação como um todo, é apresentado um conjunto de indicadores globais, pertinentes ao atendimento de metas e à 
elaboração de benchmarking, ou seja, que permita estabelecer critérios objetivos para a comparação entre edificações com características semelhantes, dentre as quais, podemos citar: eficiência energética; consumo de água eficiente; racionalização na geração de resíduos da construção civil (RDC); redução de custos de execução e de manutenção; redução de prazo de execução; minimização de ações de manutenção; aumento da vida útil; aumento da segurança dos trabalhadores e usuários. Para sua quantificação, são propostos os seguintes parâmetros, a serem calculados para o período anual: custo de execução (valor em reais, por $\mathrm{m}^{2}$ ); consumo de energia $\left(\mathrm{kWh} / \mathrm{m}^{2}\right)$ (ANSI/ASHRAE, 2013b); consumo de água $\left(\mathrm{m}^{3} /\right.$ usuário equivalente a tempo completo2) (OECD, 2008); volume de resíduos gerado $\left(\mathrm{m}^{3}\right)$; emissões de dióxido de carbono ( $\mathrm{Kg} \mathrm{CO} 2 / \mathrm{m}^{2}$ ) (OECD, 2008); taxa de demanda por manutenção (total de ocorrências); tempo dedicado a atividades de manutenção (total de horas trabalhadas); custo das atividades de manutenção (valor total, em reais); taxa de acidentes relacionados a atividades de manutenção (total de ocorrências).

Para a elaboração das avaliações, é necessário determinar, com base nas características de projeto, quais os critérios de desempenho pretendidos para os ambientes, componentes e sistemas construtivos, em função dos usos previstos e de características específicas, como, por exemplo, localização geográfica, tecnologia construtiva e níveis de vulnerabilidade social (os quais são associados a ações de vandalismo). Sendo assim, os parâmetros a serem avaliados para determinado grupo de edificações deverão ser submetidos a uma etapa inicial de validação de métricas.

São possíveis fontes para a obtenção dos dados necessários às análises: questionários, entrevistas e atividades de grupos focais; inspeção visual; medições; análise dos documentos de projeto; análise de faturas relacionadas ao consumo da edificação em operação (por exemplo, energia, água, compras); questionários; simulações e ensaios.

Para a gestão da informação da construção, é necessário que haja consistência da terminologia utilizada, tanto para a classificação dos produtos e serviços (OMNICLASS, 2012) quanto no que diz respeito às características identificadas (ABNT, 2012). O objetivo das referidas normas é permitir a padronização da terminologia dos produtos da construção civil, de modo a possibilitar a sua consulta pelos diversos agentes da cadeia construtiva. $O$ Quadro 1 apresenta um exemplo de classificação da informação e análise da recorrência de falhas, de modo a permitir o posterior rastreamento dos resultados. 7

\footnotetext{
2 Full-time equivalent - FTE (OECD, 2008).
} 


\section{Quadro 1 - Exemplo de classificação da informação e de análise de recorrência de falha}

\begin{tabular}{|c|c|c|c|c|}
\hline Item & 1 & 2 & 3 & 4 \\
\hline Elemento & $\begin{array}{c}\text { Portas de } \\
\text { acesso às salas } \\
\text { de aula }\end{array}$ & $\begin{array}{l}\text { Esquadrias de } \\
\text { fachada }\end{array}$ & Fechaduras & $\begin{array}{l}\text { Sistema de drenagem de } \\
\text { pisos (corredores) }\end{array}$ \\
\hline $\begin{array}{c}\text { Código } \\
\text { OMNICLASS } \\
\text { (OMNICLASS, } \\
\text { 2012) }\end{array}$ & $23-171115$ & $23-17131323$ & 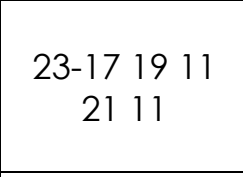 & $23-112121$ \\
\hline $\begin{array}{c}\text { Produto } \\
\text { OMNICLASS* } \\
\text { (OMNICLASS, } \\
\text { 2012) }\end{array}$ & $\begin{array}{l}\text { Porta de } \\
\text { madeira } \\
\text { genérica }\end{array}$ & $\begin{array}{l}\text { Janela com } \\
\text { caixilho em } \\
\text { perfil metálico }\end{array}$ & $\begin{array}{l}\text { Fechadura } \\
\text { para portas }\end{array}$ & Drenagem de pisos \\
\hline $\begin{array}{c}\text { Aspecto } \\
\text { crítico } \\
\text { relacionado a }\end{array}$ & componente & $\begin{array}{l}\text { sistema } \\
\text { construtivo }\end{array}$ & componente & componente \\
\hline Comentários & $\begin{array}{l}\text { Resistência } \\
\text { insuficiente da } \\
\text { folha de } \\
\text { fechamento. }\end{array}$ & $\begin{array}{c}\text { Baixa } \\
\text { resistência à } \\
\text { corrosão, } \\
\text { emperramento } \\
\text { do mecanismo } \\
\text { de abertura, } \\
\text { baixa } \\
\text { eficiência no } \\
\text { controle da } \\
\text { água, do vento } \\
\text { e dos níveis de } \\
\text { iluminação. }\end{array}$ & $\begin{array}{c}\text { Resistência } \\
\text { insuficiente } \\
\text { de } \\
\text { componente } \\
\text { s tais como } \\
\text { dobradiças } \\
\text { e } \\
\text { fechaduras. }\end{array}$ & $\begin{array}{l}\text { Caimento inadequado, } \\
\text { escoamento insuficiente. } \\
\text { Acúmulo de água em } \\
\text { corredores, áreas externas e } \\
\text { sanitários. }\end{array}$ \\
\hline $\begin{array}{l}\text { Descrição da } \\
\text { situação }\end{array}$ & $\begin{array}{l}\text { Quebras e } \\
\text { empenamento. }\end{array}$ & $\begin{array}{l}\text { Corrosão, } \\
\text { emperramento } \\
\text { de partes } \\
\text { móveis, frestas } \\
\text { entre } \\
\text { componentes. }\end{array}$ & Quebra. & Empoçamento de água. \\
\hline $\begin{array}{l}\text { Falhas } \\
\text { potenciais } \\
\text { (FRANÇA, } \\
\text { ORNSTEIN, } \\
\text { 2014) }\end{array}$ & $\begin{array}{c}\text { Resistência } \\
\text { insuficiente do } \\
\text { material } \\
\text { especificado ao } \\
\text { uso efetivo. } \\
\text { Compr. do } \\
\text { desempenho } \\
\text { esperado } \\
\text { quanto ao } \\
\text { conforto } \\
\text { térmico, } \\
\text { conforto } \\
\text { acústico, } \\
\text { estanqueidade } \\
\text { e segurança. }\end{array}$ & $\begin{array}{l}\text { Baixa eficiência } \\
\text { no controle da } \\
\text { água e do } \\
\text { vento, } \\
\text { impossibilidade } \\
\text { de controle dos } \\
\text { níveis de } \\
\text { iluminação. }\end{array}$ & $\begin{array}{c}\text { Desempenh } \\
\text { o em } \\
\text { desacordo } \\
\text { quanto à } \\
\text { durabilidade } \\
\text {. } \\
\text { Consequênc } \\
\text { ia: } \\
\text { travamento } \\
\text { e } \\
\text { movimentaç } \\
\text { ão } \\
\text { inadequado } \\
\text { s. }\end{array}$ & $\begin{array}{c}\text { Acúmulo de água em áreas } \\
\text { de circulação. Degradação } \\
\text { de elementos construtivos, } \\
\text { devido à exposição } \\
\text { frequente e prolongada à } \\
\text { água. Crítico para a } \\
\text { segurança no uso, devido } \\
\text { ao risco de escorregamento } \\
\text { e à salubridade. }\end{array}$ \\
\hline
\end{tabular}




\begin{tabular}{|c|c|c|c|c|}
\hline $\begin{array}{c}\text { Código NBR } \\
\text { 15965-2 } \\
\text { (ABNT, 2012) }\end{array}$ & $\begin{array}{l}\text { OP.60.40.23/ } \\
\text { OP.60.50.02/ } \\
\text { OP.60.50.23/ } \\
\text { OP.60.50.10 }\end{array}$ & $\begin{array}{c}\text { OP.60.70.03/ } \\
\text { OP.60.70.05/ } \\
\text { OP.60.50.08 }\end{array}$ & $\begin{array}{c}\text { OP.60.40.23/ } \\
\text { OP.60.50.02/ } \\
\text { OP.60.50.23 }\end{array}$ & OP. 50.40 .12 \\
\hline $\begin{array}{c}\text { Termo NBR } \\
15965-2 \\
\text { (ABNT, 2012) }\end{array}$ & $\begin{array}{l}\text { Resistência ao } \\
\text { ataque } \\
\text { intencional/ } \\
\text { Resistência ao } \\
\text { mal uso/ } \\
\text { Durabilidade } \\
\text { mecânica/ } \\
\text { Resistência ao } \\
\text { apodrecimento. }\end{array}$ & $\begin{array}{c}\text { Estanqueidade } \\
\text { ao ar/ } \\
\text { Penetração de } \\
\text { água/ } \\
\text { Resistência à } \\
\text { corrosão. }\end{array}$ & $\begin{array}{l}\text { Resistência } \\
\text { ao ataque } \\
\text { intencional/ } \\
\text { Resistência } \\
\text { ao mal uso/ } \\
\text { Durabilidade } \\
\text { mecânica. }\end{array}$ & Caimento. \\
\hline $\begin{array}{l}\text { Possíveis } \\
\text { causas das } \\
\text { falhas }\end{array}$ & Especificação & Execução & $\begin{array}{c}\text { Especificaçã } \\
0\end{array}$ & Execução \\
\hline Est. de caso A & 1 & 1 & 1 & 1 \\
\hline Est. de caso B & 1 & 1 & 1 & 1 \\
\hline Est. de caso C & 1 & 1 & 1 & 0 \\
\hline $\begin{array}{l}\text { Taxa de } \\
\text { recorrência } \\
(0-1)\end{array}$ & 1,00 & 1,00 & 1,00 & 0,67 \\
\hline
\end{tabular}

* Tradução nossa. Adaptado de OMNICLASS, 2012.

Fonte: acervo das pesquisadoras. Adaptado de OMNICLASS, 2012; ABNT, 2012.

O intuito da sistematização da informação proposta é possibilitar a metaavaliação das premissas de projeto. Para tanto, na Figura 2 é proposto o processo para a melhoria contínua do desempenho da edificação de tipologia construtiva semelhante, considerando a análise de indicadores de desempenho. Como se pode observar no fluxograma apresentado, os seguintes aspectos são determinantes: o atendimento às premissas de projeto, o nível de adequação aos requisitos do proprietário e o cumprimento de diretrizes ditadas pelas referências normativas, boas práticas e legislação pertinente.

A validação contínua dos resultados se faz necessária, uma vez que se pretende que as diretrizes de projeto sejam atualizadas de modo recorrente. São exemplos de modificações que representam impactos significativos na relevância dos parâmetros de avaliação: alterações em sistemas construtivos ou sistemas prediais e modificações nos usos determinados no programa de necessidades. Além disso, referências normativas, leis e boas práticas pertinentes ao projeto precisam ser permanentemente atualizadas. 


\section{Figura 2 - Processo para a consolidação de um sistema de indicadores para edificações de tipologia construtiva semelhante}

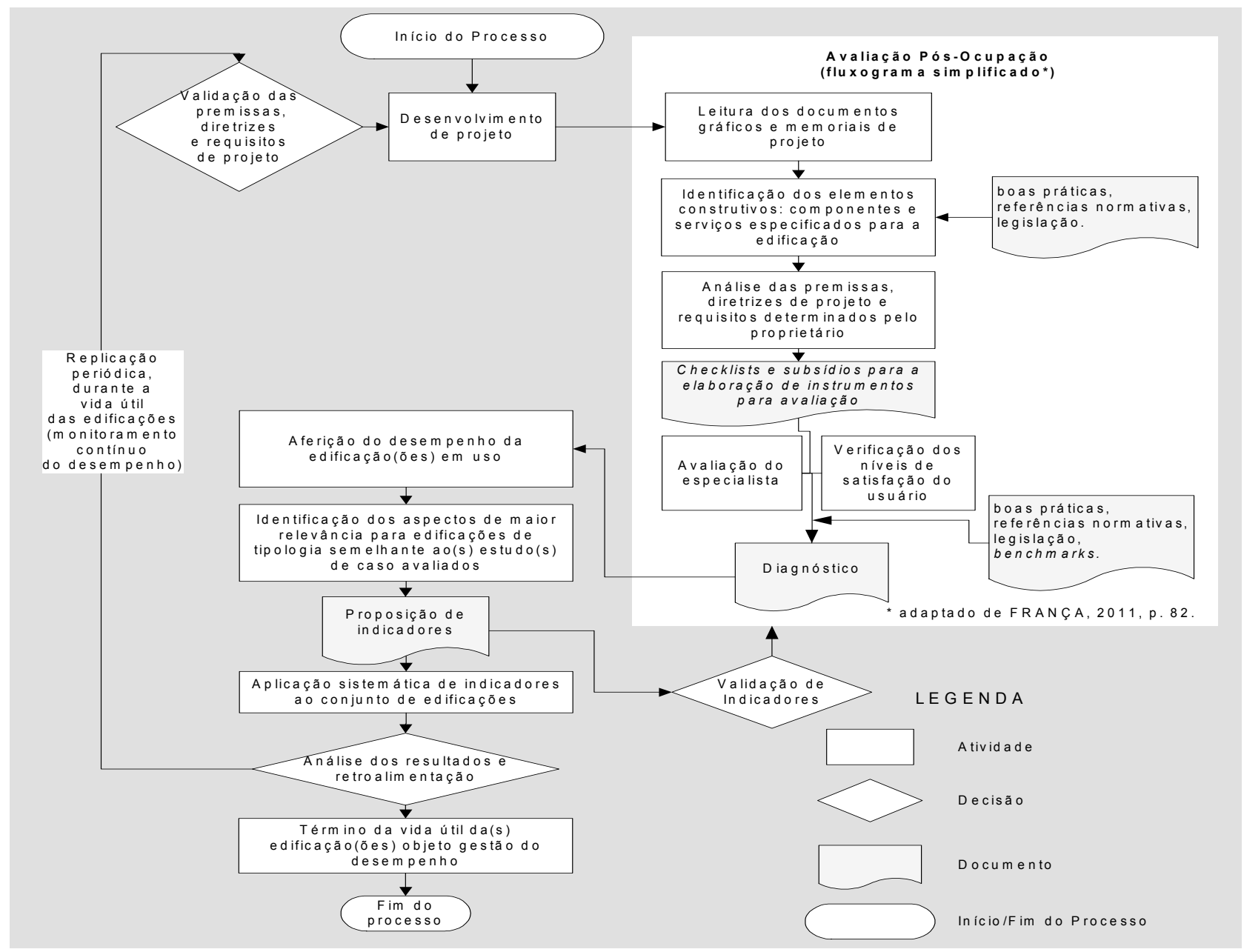

Fonte: acervo das pesquisadoras.

Para que o processo proposto possa ser implantado de modo eficiente, é necessário que exista um banco de dados comum, acessível aos responsáveis pelo planejamento, projeto, avaliação, operação e manutenção. Essa informação deve ser rastreável, de modo que os resultados estejam claramente relacionados aos componentes, serviços, sistemas construtivos e ambientes que compõem um determinado grupo de edificações.

Desta forma, responsáveis pela manutenção e auditores do desempenho da edificação devem ter acesso à informação regularmente coletada, referente às características do ambiente construído sob influência do usuário. Por sua vez, projetistas, proprietários e gestores deverão proceder à verificação do desempenho do projeto e a revisão das diretrizes (NEWBUILDINGS INSTITUTE, 2014).

\section{CONSIDERAÇÕES FINAIS}

Por meio dos métodos, técnicas e processos apresentados, se entende que é possível, com a implantação de um sistema de indicadores, aferir, de forma estruturada e continuada, o desempenho das edificações e de seus 
componentes, de modo a criar um banco dinâmico de dados dos componentes homologados, que incorpora os benefícios da experiência pregressa referente ao projeto, à construção e à ocupação de edificações de tipologia construtiva semelhante.

Essa abordagem difere da prática atual adotadas pelos gestores dos estudos de caso considerados para a pesquisa, que é baseada na determinação de diretrizes de projeto e componentes previamente homologados, sem que ocorra a validação sistemática do resultado obtido pelo conjunto de componentes e sistemas efetivamente utilizados para cada projeto, considerando as especificidades locais (tais como: localização geográfica, características climáticas e características sociais da área que irá receber a edificação).

Uma vez que as decisões de projeto são guiadas por informações, ter conhecimento acerca do desempenho real dos elementos e sistemas construtivos e suas relações é importante para que se possa obter uma edificação eficiente. A abordagem proposta permite maior clareza na apresentação de resultados, o que contribui para criar oportunidades para a flexibilização do projeto de ambientes e de componentes construtivos.

\section{REFERÊNCIAS}

ASSOCIAÇÃO BRASILEIRA DE NORMAS TÉCNICAS (ABNT). NBR 15575: Edifícios habitacionais - Parte 1: requisitos gerais. Rio de Janeiro, 2013.

NBR 5413: Iluminância de interiores. Rio de Janeiro, 1992.

NBR 10152: Níveis de ruído para conforto acústico. Rio de Janeiro, 1987.

NBR 15965-2: Sistema de classificação da informação da construção.

Parte 2: Características dos objetos da construção. Rio de Janeiro, 2012.

AMERICAN NATIONAL STANDARDS INSTITUTE/ AMERICAN SOCIETY OF HEATING, REFRIGERATING AND AIR-CONDITIONING ENGINEERS (ANSI/ASHRAE). Standard 62.1: Ventilation for Acceptable Indoor Air Quality. Atlanta, 2013.

Standard 90.1: Energy Standard for Buildings Except Low-Rise Residential Building. Atlanta, 2013.

AULICINO, P. Análise de métodos de avaliação de sustentabilidade do ambiente construído: o caso dos conjuntos habitacionais. 2008. Dissertação (Mestrado). Escola Politécnica da Universidade de São Paulo, São Paulo.

BORGES, C. A. M.; SABATTINI, F. H. O conceito de desempenho de edificações e a sua importância para o setor da construção civil no Brasil. São Paulo: Edusp, 2008.

BRE - BUILDING RESEARCH ESTABLISHIMENT. BREEAM new construction: non domestic buildings. Techinical manual. United Kingdom: BRE Global Ltd., 2011.

CARDOSO. F. F. (Coord.). Referencial técnico de certificação "Edifícios do setor de serviços - Processo AQUA". Escritórios e Edifícios escolares. São Paulo: FCAV, 2007.

CÂMARA BRASILEIRA DA INDÚSTRIA DA CONSTRUÇÃO (CBIC). Desempenho de edificações para atendimento à norma ABNT NBR 15575/2013. Câmara 
brasileira da indústria da construção. Fortaleza: Gadioli Cipolla Comunicação, 2013.

CERTIVÉA. Guide pratique du referentiel pour la qualite environnementale des bâtiments. Bâtiments tertiaires. Paris: Certivéa, 2012. Disponível em: <http://www.afilog.org/files/Referentiel_Generique_20-01-2012.pdf>. Acesso em 12 mai. 2015.

COSTA, D. B., et al. Sistema de Indicadores para benchmarking na construção civil: manual de utilização. Núcleo Orientado para a Inovação da Edificação. UFRGS: Porto Alegre, 2005.

DEGANI, C. M.. Modelo de gerenciamento de sustentabilidade de facilidades construídas. 2010. Tese (doutorado). Departamento de Engenharia Civil da Escola Politécnica da Universidade de São Paulo, São Paulo.

FEIFER, L. 786: Sustainability indicators in buildings: identifying key performance indicators. In: PLEA Proceedings 2011- 27 th Conference on Passive and Low Energy Architecture. BELGIUM, Louvain-la-Neuve: PLEA, 2011. p. 133-138.

FRANÇA, A. J. G. L.. Ambientes contemporâneos para o ensino-aprendizagem: Avaliação Pós-Ocupação aplicada a três edifícios escolares públicos, situados na Região Metropolitana de São Paulo. 2011. Dissertação (Mestrado). Faculdade de Arquitetura da Universidade de São Paulo, São Paulo..

FRANÇA, A. J. G. L., ORNSTEIN, S. W.. Técnicas de gestão da qualidade aplicadas ao diagnóstico de Avaliação Pós-Ocupação (APO). In: Encontro Nacional de Tecnologia do Ambiente Construído- ENTAC, v. 1. p. 1814-1823, 2014.

FRANÇA, A. J. G. L., ORNSTEIN, S. W.; ONO, R.. Mapas de diagnóstico: procedimentos de Avaliação Pós-Ocupação (APO) voltados à qualidade de projeto. In: 20 Simpósio Brasileiro de Qualidade do Projeto no Ambiente Construído / X Workshop Brasileiro de Gestão do Projeto na Construção de Edifícios. Rio de Janeiro: Associação Nacional de Tecnologia do Ambiente Construído / ProArq - FAU - UFRJ, v. 1. p. 297-307, 2011.

GALANEZ-ÁLVAREZ, B. Project Sponsor Group Handbook. v. 2. United Kingdom: University of Oxford, 2013.

GALVÃO, W. J. F. Roteiro para diagnóstico do potencial de reabilitação para edifícios de apartamentos antigos. 2012. Tese (Doutorado) - Faculdade de Arquitetura e Urbanismo da USP, São Paulo.

LABAKI, L. C. et al. Indicadores de eficiência energética e conforto ambiental em escolas da rede pública de Campinas, SP. In: ENCAC 2007 - IX Encontro Nacional e V Latino-americano de conforto no ambiente construído, 2007, . V. 1. p. $2112-2113$. Ouro Preto: ANTAC, 2007.

MELHADO, S. B. O plano da qualidade dos empreendimentos e a engenharia simultânea na construção de edifícios. Encontro Nacional de Engenharia de Produção - ENEGEP, 19: anais (CD-ROM). Rio de Janeiro: UFRJ/ABEPRO, 1999.

NEW BUILDINGS INSTITUTE. Key performance indicators for commercial buildings. NBI, 2014. Disponível em: <http://newbuildings.org/sites/default/files/ What_is_a_KPI_1.pdf>. Acesso em: 20 mar. 2015. 
ORGANIZATION FOR ECONOMIC COOPERATION AND DEVELOPMENT (OECD). OECD Core set of indicators for environmental performance reviews. A synthesis report by the Group on the State of the Environment. Environment Monographs, n.83, 1993. Disponível em: <http:// http://indicators.ucdavis.edu/waf/biblio/ oecd-core-set-indicators-environmental-performance-reviews-synthesis-reportgroup-state>. Acesso em: 10 mar. 2015.

OECD Glossary of statistical therms. OECD, 2008. Disponível em: <http:// stats.oecd.org/glossary/>. Acesso em: 10 mar. 2015.

OMNICLASS. Table 23 - Products. OMNICLASS, 2012. Disponível em: <http:// Www.omniclass.org>. Acesso em: 11 jun. 2015.

SILVA, V. G. Indicadores de sustentabilidade de edifícios: estado da arte e desafios para desenvolvimento no Brasil. Revista Ambiente Construído. Porto Alegre: ANTAC. v. 7, n. 1. p. 47-66, 2007.

UNITED STATES GREEN BUILDING COUNCIL (USGBC). LEED 2009 Reference Guide for New Construction and Major Renovation. USGBC, 2009.

VAN DER VOORDT, T. JM, VAN WEGEN, H. BR. Architecture in use: an introduction to the programming, design and evaluation of buildings. The Neederlands: THTHO Publishers, 2005. 